\title{
The Application of Incentive Mechanisms for the Participation of Enterprises in Collaborative Networks from an Economic Perspective
}

\author{
Hendrik Jähn \\ Chemnitz University of Technology, Dept. of Economic Sciences, Thüringer Weg 7 \\ 09126 Chemnitz, Germany \\ hendrik.jaehn@wirtschaft.tu-chemnitz.de
}

\begin{abstract}
This contribution is to be assigned to the operative production management of collaborative networks. By the application of incentive mechanisms, a competitive and effective management of production processes allows sustainable success. Incentives are relevant for all stages of a network lifecycle. In this paper, the problem is discussed that enterprises from a pool of cooperationwilling enterprises do not take part in a certain value-added process, even though it would be possible from the technical point of view and would also be economically reasonable. As a reason for this behavior, the utility calculus of an enterprise plays a decisive role. To convince these enterprises to collaborate in a value-added process, primarily incentive mechanisms are to be applied. In the following, this topic is taken on and is discussed extensively by means of two concrete examples. In this connection, a concrete calculation model both for the amount of incentives and the profit share is introduced.
\end{abstract}

Keywords: Collaborative network, incentive mechanisms, operative network management, sustainable success.

\section{Motivation}

The implementation of value-added processes within networked production structures has strongly gained in importance. Integral advantages are a high degree of flexibility, sustainability and cost saving potential. However, not in every case value-added processes may be realized by collaboration although this would be reasonable from an economic perspective. So, the effort of an enterprise to cooperate primarily depends on the economic advantageousness of a project, i.e. when the utility can be maximized or a benefit can be achieved respectively. A project is, however, only reasonable if the overall benefit of the network is maximized or if a benefit results. In certain cases, enterprises do maximize their benefit without cooperation although participation is desirable if seen as a whole. Reasons for not cooperating may principally be found in a shortage of production capacity or missing financial attractiveness of an order. In these cases, the network management has the possibility to interfere in a regulating way. To achieve a sustainable outcome, incentive mechanisms can be applied to a certain degree for selected enterprises. 


\section{Incentives in an Economic Environment}

The application of incentives in an economic environment is possible in various ways and for numerous situations. In relation to the analysis of principal-agent-relations [1] within the frame of the principal-agent-theory [2], [3], incentives can be applied as a possible solution approach for problems classes representing hidden action and hidden information. Here, the whole enterprise network represents the principal; the involved enterprises are the agents. In this context, incentives serve as alignment of interests between principal and agent after conclusion of a contract. At this point, a reduction or removal respectively of the moral hazard on the agent's side is achieved. Within the frame of the principal-agent-theory, these actions are tendentially agentrelated. Here, incentive systems represent the combination of several single incentives. In this context, incentives also stand for a suitable measure for an alignment of interests of the agent to those of the principal [4].

In this context, incentives are understood as a situational condition, which is supposed to motivate the economic agents due to their individual needs, i.e. utility structure for a performance for the purpose of the principal. By means of incentives, agents are influenced to perform actions in the intensity desired by the principal (management) [5]. Incentive systems represent the sum of all working conditions designed purposely enforcing certain behavior patterns and reducing the probability of the occurrence of undesirable behavior patterns. Behavior patterns are made stronger by the application of positive incentives in form of rewards taken as an example. Undesirable behavior can be reduced by negative incentives in form of sanction mechanisms [4]. From that results the so-called "motivation problem", which in its core statement says that an individual needs an incentive to produce a certain performance or to omit an action as long as this has no connection to the individual utility maximization [6]. By means of incentives, it is aspired to suppress self-interested behavior of agents and to adjust their behavior to the aims of the principal. However, this method does not lead to the fact that an agent does not maximize its individual utility, but it is rather attempted to bring the agent to actions by directed incentives for which, by maximizing of his individual utility, also the utility of the principal is maximized. In this connection, one also speaks of incentive compatibility [4]. This procedure can be summarized by the terms interest alignment or interest harmonization. In addition to these rather generally formulated functions of incentive systems, incentives can also be divided into single part functions [7]:

- Activation function: stimulation of motivation and cognitive abilities,

- Controlling function: influence of the agents' behavior,

- Information function: agents get signals regarding desirable behavior,

- Selection function: principals get information about agents' performance and

- Coordination function: coordination of single actions of agents.

To ensure that these functions are fulfilled as good as possible, incentive systems shall meet different requirements. On the one hand, incentives need to be economically justifiable. Also the application of incentive mechanisms is only reasonable if the costs caused by the incentive system are lower than resulting (additional) earnings. These costs result from the measures conducted for the incentive system and also the administrative costs. 


\section{Incentive Design with insufficient Production Capacity}

To be able to guarantee and quantify incentives, reasons for this need to be identified. So, not taking part in a value-added process may for an individual enterprise be the best alternative, but not necessarily for the whole network. This would be the case if a core competency is missing in the value-added chain to be able to perform a service in a collaborative network. In such a case the "missing" enterprise needs to be guaranteed suitable incentives for participating. In the following it is assumed that an enterprise does not cooperate due to insufficient production capacity (machine or personnel). Here incentives need to be paid for supplying additional capacities.

There are several approaches to solve this problem. One was is to compensate the additional costs for the enterprise supplying additional capacities. The starting point is that an enterprise is not able to fulfill the respective requirements within the given time frame on the basis of a request of the network. As a reason for this it is assumed that insufficient production capacities lead to this decision. Although a possible completion date can be stated within the frame of the tender preparation, this should however be oriented at the desired date of the customer or, in case the customer does not give a desired date, the date should be within a reasonable time span for the fulfillment of an order. At this point, it must be decided whether the enterprise being the only one offering the demanded core competence can be made giving a (realistic) offer. A practicable solution would be, taken as an example, to create suitable capacities by replanning. This option marks the starting point for the following considerations.

It is assumed that a certain enterprise aspires to be able to give an offer by suitable replanning. The costs accruing by replanning are difficult to quantify, which however is indispensable for the calculation of the incentives. Personnel costs resulting from the replanning taken as an example may be included in the costs of replanning. Additional costs resulting from overtime, nightshifts or weekend work can be included as well. Furthermore, costs accruing from delayed delivery caused by other replanned orders have to be calculated. These additional accruing costs need to be refunded to the affected enterprise, which can be interpreted as an incentive payment. Here, the affected enterprise has to raise a part of the incentive amount by itself. This is explained by the fact that the respective enterprise is entitled to get a share in profit when taking part in the value-added process, which would not be obtained when not participating.

Guaranteeing incentive payments is based on the assumption that all enterprises aspire individual utility maximization. So, it is always first goal to cooperate in a value-added process as long as benefit (profit) can be obtained. For the case that a customer order cannot be realized due to missing enterprises, no benefit can be achieved. As a general rule, abandoning the participation in a value-added process by an enterprise has high probability if the missed benefit is lower than additional costs. These include replanning costs or further costs for capacity expansion. The latter kind of costs includes costs for the purchasing or leasing of a machine, but also additional personnel costs like overtime payments or rewards for working on holidays or at the weekend. Such costs have to be taken into account for consideration of total costs for the calculation of possible incentives. 


\section{Incentive Design for Lacking Financial Rewarding of an Order}

\subsection{Description of the Situation}

The financial aspect of an order is primarily represented by the profit to be gained for an enterprise. So the focus is laid on approaches for calculating enterprise-related profit shares $g_{i}$. For this purpose various approaches have been developed [8] and are available. As a precondition, knowledge concerning the entire distributable profit $G$ is essential. If no quote can be submitted for the concrete enquiry of a customer in the network from the network's side, this situation is unsatisfactory for all involved parties. Therefore, an alternative solution needs to be found.

In the present case, it is assumed that the enterprise(s), which need(s) to be engaged for the considered value-added process possess(es) sufficient production capacity. So, the missing financial profitability of the order can be considered to be the only reason for the rejection. Therefore, it is the aim to convince the respective enterprises (here it is assumed that only one enterprise $i^{*}$ is concerned) regarding participation in the value-added process by means of suitable incentives. To be able to make a decision for or against an incentive payment, various results connected to the guaranteeing or omission of incentives, need to be analyzed and compared.

Based on the situation that the single shares of profit $g_{i}$ were determined within the frame of an algorithm for determining the profit $G$, but no agreement could be found with enterprise $i^{*}$ regarding profit $g_{i^{*}}$ and therefore it was not possible to submit a quote for a customer enquiry, suitable incentives need to be guaranteed. Quantification of these incentives is described in the following. For this purpose, an algorithm for the calculation of profit and incentive payments within networked production structures is introduced.

Assuring a minimal profit for an enterprise represents a material incentive on the network's side. This incentive is guaranteed in cases if an enterprise rejects participation due to lacking profitability, although the respective core competency is urgently needed to be able to implement the value-added process in the network. This situation always occurs when the share of profit of an enterprise is lower than the expectation of profit (minimal profit) according to the model for distribution of profit. So, a guaranteed minimal profit is a security incentive.

\subsection{Calculation of the Individual Share of Profit of an Enterprise}

Assumptions. The calculation of the cash flows in the network may be conducted for a comprehensive model regarding the distribution of profit when applying a suitable calculation approach. For the modeling of this approach regarding determining participation incentives, the following five assumptions apply representing the general conditions for the application of incentive mechanisms:

a) guaranteed incentive payments are paid by integration into the process of distribution of profits,

b) each enterprise receives an individual share of profit $g_{i}$, which depends on the part in total costs and also consists of a fixed part,

c) all incentive and profit payments ( $a_{i}$ or $g_{i}$ respectively) resulting from the concrete value-added process have to be financed from the realized network profit $G$ of the considered value-added process, 
d) the model for distribution of profit can be modified by agreements about minimal profit shares between network and the single enterprises; the minimal profit share of an enterprise $g_{i}{ }^{\min }$ has to be guaranteed by the regular share of profit and if necessary by an incentive payment.

Modeling. Consecutively the modeling of the calculation approach is introduced. This calculation approach can then be applied if a finite number of enterprises take part in the value-added process, but only one enterprise receives incentive payments.

Condition 1: The sum of the profit share of the enterprises $g_{i}$ results from the gained or distributable network profit $G$ less all guaranteed incentive payments $a_{i}$. This side condition implies that the guaranteed incentive payments $a_{i}$ in the present model are financed from profit $G$.

Condition 2: The second condition contains the calculation regulation for the share of profit of enterprises $g_{i}$. In the present case, a two-component-approach is applied with a distribution parameter according to [8]. The profit share consists of a fixed and a variable part. Both parts are calculated according to the respective formulas.

Condition 3: The guaranteed minimal profit of an enterprise $g_{i}{ }^{\text {min }}$ consists of the individual realized share of profit of this enterprise $g_{i}$ and an incentive payment being possibly guaranteed to enterprise $a_{i}$. In addition, a difference variable $u_{i}$ is introduced. Its function is to represent a potential difference between the individual share of profit $g_{i}$ and the guaranteed minimal profit $g_{i}{ }^{m i n}$. This can occur if the individual share of profit is higher than the guaranteed minimal profit because no incentive payment is necessary. Therefore, variable $u_{i}$ makes sure that the equation condition is valid also in such cases. Each enterprise represents a specific $u_{i}$.

Condition 4: This condition is based on the third condition. So, variable $u_{i}$ is only applied for cases in which no incentive payment $a_{i}$ is guaranteed to the respective enterprise. This results in the fact that in this case $u_{i}$ gets a value of not equal to zero if the incentive payment $a_{i}$ equals zero and vice versa. Therefore, the product from difference variable $u_{i}$ and incentive payment $a_{i}$ has to be zero in each case.

Additional conditions: For completion of the modeling several more model conditions need to be determined. This also contains the respective definition and co domains of the variables. So, non-negativity conditions for the total profit $G$, the incentive payments $a_{i}$, the individual share of profit $g_{i}$, the guaranteed individual minimal profits $g_{i}^{\min }$, the individual $\operatorname{costs} c_{i}$ and the difference variable $u_{i}$ have to be introduced. Furthermore, it has to be stated that the weighting parameter $\alpha$ shall be between 0 and 1, as the incentive is supposed to be applied independently. Finally, the number of enterprises in the network $n$ has to be defined.

Calculation Approach. In the following, the previously described calculation approach is presented formally. For this modeling, it needs to be noted that only a selected model for distribution of profit according to condition 2 is applied. The calculation approach consists of four equations and three unknown variables $a_{i}, g_{i}, u_{i}$. This constellation makes an unproblematic solving of the task possible. However, the complexity of the model increases with a rising number of enterprises taking part in the value-added process, as then $n+3$ equations need to be solved with $n+2$ unknown variables. 


\begin{tabular}{|c|c|}
\hline Condition 1: & $\sum_{i=1}^{n} g_{i}=G-\sum_{j=1}^{n} a_{i}$ \\
\hline Condition 2: & $g_{i}=\alpha \cdot \frac{\sum_{j=1}^{n} g_{i}}{n}+(1-\alpha) \cdot \frac{c_{i}}{\sum_{j=1}^{n} c_{i}} \cdot \sum_{j=1}^{n} g_{i}$ \\
\hline Condition 3: & $g_{i}^{\min }=g_{i}+a_{i}-u_{i}$ \\
\hline Condition 4: & $a_{i} \cdot u_{i}=0$ \\
\hline
\end{tabular}

Fig. 1. Calculation model for incentives

Example. The calculation approach introduced in the section before can be applied for cases, in which a finite number of enterprises $n$ takes part in a value-added process and only one enterprise receives a financial incentive. Having a number of three enterprises in the network, when taken as an example and only one enterprise receiving an incentive, the following six equations result including five unknown variables $a_{i}, g_{1}, g_{2}, g_{3}, u_{i}$.
(1) $\sum_{j=1}^{n} g_{i}=G-a_{1}$
(2) $g_{1}=\alpha \cdot \frac{\sum_{j=1}^{n} g_{i}}{n}+(1-\alpha) \cdot \frac{c_{1}}{\sum_{j=1}^{n} c_{i}} \cdot \sum_{j=1}^{n} g_{i}$
(3) $g_{2}=\alpha \cdot \frac{\sum_{j=1}^{n} g_{i}}{n}+(1-\alpha) \cdot \frac{c_{2}}{\sum_{j=1}^{n} c_{i}} \cdot \sum_{j=1}^{n} g_{i}$
(4) $g_{3}=\alpha \cdot \frac{\sum_{j=1}^{n} g_{i}}{n}+(1-\alpha) \cdot \frac{c_{3}}{\sum_{j=1}^{n} c_{i}} \cdot \sum_{j=1}^{n} g_{i}$
(5) $g_{1}^{\text {min }}=g_{1}+a_{1}-u_{1}$
(6) $a_{1} \cdot u_{1}=0$

Fig. 2. Calculation model for incentives for three network participants 
In the following a quick solution finding is demonstrated. Here enterprise 1 was assumed to receive incentive payments. Consideration of this condition in the model leads to the result that the difference variable $u_{l}$ is zero. It follows:

$$
\begin{aligned}
& \text { (6) } a_{1} \cdot u_{1}=0 \quad a_{1} \neq 0 \rightarrow u_{1}=0 \\
& \text { (5) } g_{1}^{\text {min }}=g_{1}+a_{1}
\end{aligned}
$$

After that, the formula for a calculation of the profit share has to be created.

$$
\begin{aligned}
& \text { (1) } \sum_{j=1}^{n} g_{i}=G-a_{1} \\
& \text { (2) } g_{1}=\alpha \cdot \frac{\sum_{j=1}^{n} g_{i}}{n}+(1-\alpha) \cdot \frac{c_{1}}{\sum_{j=1}^{n} c_{i}} \cdot \sum_{j=1}^{n} g_{i} \\
& \text { (1) in }(2) \rightarrow\left(2^{\prime}\right): \quad g_{1}=\alpha \cdot \frac{G-a_{1}}{n}+(1-\alpha) \cdot \frac{c_{1}}{\sum_{j=1}^{n} c_{i}} \cdot\left(G-a_{1}\right)
\end{aligned}
$$

Then, equation (2') can be included in equation (5).

$$
\left(2^{\prime}\right) \text { in }(5) \rightarrow\left(5^{\prime}\right): \quad g_{1}^{\min }=\alpha \cdot \frac{G-a_{1}}{n}+(1-\alpha) \cdot \frac{c_{1}}{\sum_{j=1}^{n} c_{i}} \cdot\left(G-a_{1}\right)+a_{1}
$$

For calculation of the amount of incentive payment $a_{1}$, equation ( $5^{\prime}$ ) has to be transposed to $\mathrm{a}_{1}$. To facilitate this process, the following two substitutions are conducted:

$$
\begin{aligned}
& (1-\alpha)=\beta \\
& \frac{c_{1}}{\sum_{i=1}^{n} c_{i}}=m_{1}
\end{aligned}
$$

So, transposition from equation ( $\left.5^{\prime}\right)$ to $a_{l}$ can be realized as follows:

$$
\begin{gathered}
g_{1}^{\text {min }}=\alpha \cdot \frac{G-a_{1}}{n}+\beta \cdot m_{1} \cdot\left(G-a_{1}\right)+a_{1} \\
g_{1}^{\text {min }}=a_{1}+\left(G-a_{1}\right) \cdot\left(\frac{\alpha}{n}+\beta \cdot m_{1}\right) \\
g_{1}^{\text {min }}=a_{1}+G \cdot\left(\frac{\alpha}{n}+\beta \cdot m_{1}\right)-a_{1} \cdot\left(\frac{\alpha}{n}+\beta \cdot m_{1}\right) \\
g_{1}^{\text {min }}-G \cdot\left(\frac{\alpha}{n}+\beta \cdot m_{1}\right)=a_{1}-a_{1} \cdot\left(\frac{\alpha}{n}+\beta \cdot m_{1}\right) \\
g_{1}^{\text {min }}-G \cdot\left(\frac{\alpha}{n}+\beta \cdot m_{1}\right)=a_{1} \cdot\left(1-\left(\frac{\alpha}{n}+\beta \cdot m_{1}\right)\right) \\
a_{1}=\frac{g_{1}^{\text {min }}-G \cdot\left(\frac{\alpha}{n}+\beta \cdot m_{1}\right)}{1-\left(\frac{\alpha}{n}+\beta \cdot m_{1}\right)} \\
a_{1}=\frac{G \cdot\left(\frac{\alpha}{n}+\beta \cdot m_{1}\right)-g_{1}^{\text {min }}}{\frac{\alpha}{n}+\beta \cdot m_{1}-1}
\end{gathered}
$$

Finally, the variables inserted by substitution can be replaced by the original terms again. The following formula results from that and by means of it, the amount of the incentive payment to enterprises 1 can be calculated: 


$$
a_{1}=\frac{G \cdot\left(\frac{\alpha}{n}+(1-\alpha) \cdot \frac{c_{1}}{\sum_{j=1}^{n} c_{i}}\right)-g_{1}^{\min }}{\frac{\alpha}{n}+(1-\alpha) \cdot \frac{c_{1}}{\sum_{j=1}^{n} c_{i}}-1}
$$

In addition to the realized individual share of profit $g_{l}$, enterprise 1 receives an incentive payment $a_{1}$. Consecutively the remaining profit shares can be calculated by means of equations (2), (3) and (4). Finally, all necessary values can be calculated.

\section{Conclusions}

In the previous sections, it has been proven that the implementation of incentive mechanisms is an effective and promising instrument of the operative network controlling. For this purpose the maximization of the profit both for the network (principal) and for the single enterprises (agents) is realized by guaranteeing financial incentives. Possible criteria for the assessment of a contractual behavior of the enterprises are comparisons between target and actual performance when considering various performance parameters [9]. The provision of service according to the contractual duty has an essential importance for the network as these criteria have a considerable influence on customer satisfaction and decide on the continuance and sustainable success. Because of its structure the model is applicable for an unlimited number of network participants. In case a different approach for profit distribution is considered, the affected side conditions need to be modified.

\section{References}

1. Ross, S.A.: The Economic Theory of Agency: The Principal's Problem. American Economic Review 63, 134-139 (1973)

2. Furubotn, E.G., Richter, R.: Institutions and Economic Theory: The Contribution of the New Institutional Economics, 2nd edn. University of Michigan Press, Ann Arbor (2005)

3. Laffont, J.J., Martimort, D.: The Theory of Incentives - The Principal-Agent-Model. Princeton University Press, Princeton (2002)

4. Picot, A., Dietl, H., Franck, E.: Organisation - Eine ökonomische Perspektive, 4th edn. Schäffer-Poeschel, Stuttgart (2005)

5. Macho-Stadler, I., Pérez-Castrillo, D.: An Introduction to the Economics of Information Incentives and Contracts, 2nd edn. Oxford University Press, Oxford (2001)

6. Milgrom, P., Roberts, J.: Economics, Organization and Management. Prentice Hall, Englewood Cliffs (1992)

7. Winter, S.: Prinzipien der Gestaltung von Managementanreizsystemen. Gabler, Wiesbaden (2002)

8. Jähn, H., Fischer, M., Teich, T.: Distribution of Network generated Profit by considering individual Profit Expectations. In: Camarinha-Matos, L.M., et al. (eds.) Establishing the Foundation of Collaborative Networks, pp. 337-344. Springer, New York (2007)

9. Jähn, H.: Value-added process-related performance analysis of enterprises acting in cooperative production structures. Production Planning \& Control 20, 178-190 (2009) 